\title{
Expression and purification of recombinant proteins based on human prostate stem cell antigen and heat shock protein-70
}

\author{
LEI DONG $^{1^{*}}$, XIAOPENG ZHANG $^{2 *}$, CHANGMING YU ${ }^{2}$, JUN REN $^{2}$, \\ LIHUA HOU ${ }^{2}$, LING FU' ${ }^{2}$, SHAOQIONG YI ${ }^{2}$ and WEI CHEN ${ }^{2}$ \\ ${ }^{1}$ Clinical Laboratory Center, PLA Air Force General Hospital, Haidian, Beijing 100142; \\ ${ }^{2}$ Beijing Institute of Biotechnology, Academy of Military Medical Sciences, Fengtai, Beijing 100071, P.R. China
}

Received December 1, 2012; Accepted February 5, 2013

DOI: $10.3892 / \mathrm{etm} .2013 .967$

\begin{abstract}
The aim of this study was to express and purify recombinant proteins based on human prostate stem cell antigen (PSCA) and heat shock protein-70 (HSP70). The PSCA gene and various structural domains of HSP70 were amplified by polymerase chain reaction (PCR) with the respective primers. Then, the PSCA was cloned into the prokaryotic expression vector pET21a(+) with the amino-terminus, carboxyl-terminus and overall length of HSP70, by enzyme digestion to construct the recombinant plasmids pET21-PSCA-HSPN, pET21PSCA-HSPC and pET21-PSCA-HSP, respectively. After being expressed in Escherichia coli (E. coli) by isopropyl $\beta$-D-1-thiogalactopyranoside (IPTG) induction, recombinant fusion proteins were purified. Western blotting was performed to confirm the expression of the recombinant proteins. The results revealed that recombinant plasmids were successfully constructed. The PSCA-HSPC and PSCA-HSP expressed in E. coli existed in soluble form, as confirmed by sodium dodecyl sulfate-polyacrylamide gel electrophoresis (SDS-PAGE). The purity of the recombinant proteins PSCA-HSPC and PSCA-HSP reached $>95 \%$ following purification with the nickel-nitrilotriacetic acid (Ni-NTA) resin, Phenyl-Sepharose Fast Flow and Superdex 75, which lays a foundation for the development of vaccines for prostate cancer.
\end{abstract}

\section{Introduction}

Prostate cancer is the most common non-cutaneous cancer with a high mortality rate in American males. Previously,

Correspondence to: Dr Shaoqiong Yi or Dr Wei Chen, Beijing Institute of Biotechnology, Academy of Military Medical Sciences, 20 Dongdajie, Fengtai, Beijing 100071, P.R. China

E-mail: yishaoqiong@163.com

E-mail: chenwei_0226@126.com

*Contributed equally

Key words: prostate stem cell antigen, heat shock protein, structural domain, recombinant fusion protein studies on novel anticancer strategies have introduced prostate cancer immunotherapy, which represents a highly attractive therapeutic strategy for cancer treatment. Prostate stem cell antigen (PSCA), first described by Reiter et al, is a surface glycoprotein and has a $30 \%$ homology with stem cell antigen-2 (1). PSCA is expressed in $85 \%$ of prostate cancer specimens with high tissue specificity. There is a direct correlation between the expression level of PSCA and tumor stage, grade and bone metastasis (2). To date, studies have confirmed that vaccination based on PSCA enhances the cellular and humoral immune responses and inhibits the growth of PSCAexpressing tumors in mice (3-5). Therefore, PSCA may be a potential target for prostate cancer immunotherapy.

Heat shock protein-70 (HSP70) is a major molecular chaperone, which assists in transport, folding and assembly of proteins in the cytoplasm transmembrane. It is also involved in the functions of the mitochondria, endoplasmic reticulum and nucleus. Previous studies have demonstrated that vaccination with HSP70-peptide complexes elicit specific antitumor responses (6-9). These findings suggest that HSP70 is involved in the process of antigen presentation and has potential as a chaperone for specific antigens in vaccines.

In the present study, we successfully constructed recombinant plasmids based on PSCA and HSP70 and obtained soluble recombinant proteins with a purity $>95 \%$, which lays the foundation for the development of a vaccine for prostate cancer.

\section{Materials and methods}

Main reagents. The plasmids pET $21 \mathrm{a}(+)$, pMD-HSP70 and pMD-PSCA were stored in the Beijing Institute of Biotechnology, Academy of Military Medical Sciences. Escherichia coli (E. coli) DH5 $\alpha$ and BL21(DE3), pMD18-T, LA Taq, T4 DNA ligase, DNA marker 2000 and the restriction enzyme were purchased from Takara Biotechnology Co., Ltd. (Dalian, China). A plasmid mini kit was purchased from Promega Biotech Co., Ltd. (Beijing, China). Anti-PSCA polyclonal antibody and fluorescein isothiocyanate (FITC)conjugated goat anti-rabbit IgG antibody were purchased from Santa Cruz Biotechnology, Inc. (Santa Cruz, CA, USA). Purification medium was purchased from Qiagen (Beijing, China). All other domestic reagents used in this study were 
of analytical grade. The study was approved by the ethics committee of the Academy of Military Medical Sciences (Beijing, China).

Construction of expression plasmids. All constructions were cloned into the pET21a(+) vector. The human PSCA gene was amplified from the pMD-PSCA vector with the primers 5'-CCG CAT ATG TGC TAT AGC TGC AAA GCC-3' and 5'-CCG GAA TTC CAG GGC ATG GGC CCC GCT-3' and cloned into the $N d e I$ and $E c o$ RI sites of pET21a(+) to generate pET21-PSCA. The amino-terminus, carboxyl-terminus and overall length genes of HSP70 were amplified from the pMD-HSP70 vector with the primers 5'-CCG GAA TTC ATG GCC AAA GCC GCG GCG-3' and 5'-CCG CTC GAG TCG CTT GTT CTG GCT GAT-3', 5'-CCG GAA TTC CTG AAC AAG AGC ATC AAC-3' and 5'-CCG CTC GAG ATC TAC CTC CTC AAT GGT-3', 5'-CCG GAA TTC ATG GCC AAA GCC GCG GCG-3' and 5'-CCG CTC GAG ATC TAC CTC CTC AAT GGT-3', respectively. Then, amplification products of various structural domains of HSP70 were cloned into the EcoRI and XhoI sites of pET21-PSCA to generate pET21PSCA-HSPN, pET21-PSCA-HSPC and pET21-PSCA-HSP. PSCA amplification was performed for $3 \mathrm{~min}$ at $94^{\circ} \mathrm{C}$, immediately followed by $30 \mathrm{sec}$ at $94^{\circ} \mathrm{C}, 30 \mathrm{sec}$ at $55^{\circ} \mathrm{C}$ and $30 \mathrm{sec}$ at $72^{\circ} \mathrm{C}$ for 30 cycles. HSPN, HSPC and HSP were incubated for $3 \mathrm{~min}$ at $94^{\circ} \mathrm{C}$, followed by $30 \mathrm{sec}$ at $94^{\circ} \mathrm{C}, 30 \mathrm{sec}$ at $55^{\circ} \mathrm{C}$ and $90 \mathrm{sec}$ at $72^{\circ} \mathrm{C}$ for 30 cycles, respectively. An additional extension step was performed for $10 \mathrm{~min}$ at $72^{\circ} \mathrm{C}$. DNA sequencing was performed to confirm that all constructs had the desired sequence and open reading frame. All plasmids were transformed into DH5 $\alpha$-competent $E$. coli. Plasmid DNA copies were amplified in liquid culture and purified using a plasmid mini kit.

Expression of recombinant fusion proteins. A single transformed BL21(DE3) colony was inoculated into $10 \mathrm{ml}$ Luria-Bertani (LB) medium supplemented with ampicillin $(100 \mathrm{~g} / \mathrm{ml})$ followed by agitation at $250 \mathrm{rpm}$ overnight at $37^{\circ} \mathrm{C}$. Then, $3 \mathrm{ml}$ culture was transferred to $300 \mathrm{ml}$ fresh LB medium in a $1,000 \mathrm{ml}$ shake flask. The culture was grown at $37^{\circ} \mathrm{C}$ with $250 \mathrm{rpm}$ agitation until the optical density at $600 \mathrm{~nm}$ (OD600) reached 1.2. Then, induction was performed with the addition of $0.3 \mathrm{ml} 1 \mathrm{M}$ isopropyl $\beta$-D-1-thiogalactopyranoside (IPTG). At $5 \mathrm{~h}$ after induction, a $1 \mathrm{ml}$ sample was collected.

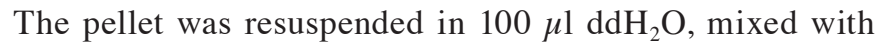
$2 \mathrm{X}$ sodium dodecyl sulfate (SDS) loading buffer [0.0625 M Tris- $\mathrm{HCl}$ (pH 6.8), 2\% SDS, 25\% glycerol, 5\%-mercaptoethanol and $0.01 \%$ bromphenol blue] and heated at $95^{\circ} \mathrm{C}$ for $4 \mathrm{~min}$. Following centrifugation at $10,000 \mathrm{x}$ g for $5 \mathrm{~min}, 20 \mu \mathrm{l}$ supernatant was analyzed by SDS-polyacrylamide gel electrophoresis (PAGE) and stained by Coomassie blue R-250.

Lysis of cells and purification of the recombinant fusion proteins. Following induction for $5 \mathrm{~h}$, cells were harvested by centrifugation at $10,000 \mathrm{x}$ g for $15 \mathrm{~min}$ from 4,000 $\mathrm{ml}$ culture, resuspended in $100 \mathrm{ml} 20 \mathrm{mM}$ lysis buffer $\left(20 \mathrm{mM} \mathrm{NaH}_{2} \mathrm{PO}_{4}\right.$, $0.5 \mathrm{mM} \mathrm{NaCl}$ and $10 \mathrm{mM}$ imidazole; $\mathrm{pH}$ 7.4) and lysed with a high pressure homogenizer (pressure, 900 bar; one time). The supernatant collected following centrifugation at $15,000 \mathrm{x} g$ for 20 min was loaded in a nickel-nitrilotriacetic acid (Ni-NTA) resin column pre-equilibrated with $20 \mathrm{mM}$ lysis buffer. The column was washed with equilibration buffer and when the absorbance at $280 \mathrm{~nm}$ was $<0.01$, the column was eluted using $50 \mathrm{mM}$ imidazole in $20 \mathrm{mM}$ equilibration buffer followed by a linear gradient of 50-500 mM imidazole in $20 \mathrm{mM}$ equilibration buffer. The ammonium sulfate powder was added to the above elution liquid containing the eluted target protein to a concentration of $1 \mathrm{M}$. The above elution was loaded in to the Phenyl-Sepharose Fast Flow (FF) column pre-equilibrated with $20 \mathrm{mM}$ phosphate-buffered saline (PBS; pH 7.4) supplemented with $1 \mathrm{M}$ ammonium sulfate. The column was washed with equilibration buffer and when the absorbance at $280 \mathrm{~nm}$ was below 0.01 , the column was eluted using a linear gradient of 1-0 M ammonium sulfate in $20 \mathrm{mM}$ phosphate buffer ( $\mathrm{pH}$ 7.4). The elution peak from the Phenyl-Sepharose FF column was loaded in to the HiLoad ${ }^{\mathrm{TM}}$ 26/60 Superdex 75 and washed with $20 \mathrm{mM}$ phosphate buffer ( $\mathrm{pH} 7.4$ ); then the target protein peak was collected. The purified recombinant proteins were collected and analyzed by SDS-PAGE analysis. The final purity of products was determined by means of size exclusion-high-performance liquid chromatography (SE-HPLC).

SDS-PAGE analysis. SDS-PAGE was performed using $12 \%$ resolution gel on the MiniProtean 3 system (Bio-Rad, Hercules, CA, USA). Briefly, the whole $E$. coli cell lysate was loaded on the gel. Then, the gel was electrophoresed at $150 \mathrm{~V}$ for $1 \mathrm{~h}$ and stained with Coomassie brilliant blue R-250. Low molecular weight protein markers were rabbit phosphorylase B (97.2 kDa), bovine serum albumin $(66.4 \mathrm{kDa})$, rabbit actin (44.3 kDa), bovine carbonic anhydrase (29.0 kDa), soybean trypsin inhibitor $(20.1 \mathrm{kDa})$ and hen egg white lysozyme (14.3 kDa).

Identification of recombinant fusion proteins with western blotting. Following electrophoresis at $150 \mathrm{~V}$ for $1 \mathrm{~h}$, the target protein was transferred to a polyvinylidene difluoride (PVDF) membrane using a Bio-Rad semidry apparatus with transfer buffer [10 mM CAPS buffer ( $\mathrm{pH} 11), 10 \%$ methanol]. The PVDF membrane, wetted in $100 \%$ methanol, was soaked in transfer buffer before being placed on the PAGE gel. After being coated with anti-PSCA monoclonal antibody, the membrane was incubated at $4^{\circ} \mathrm{C}$ for $24 \mathrm{~h}$. Then, it was washed and blocked with blocking buffer [3\% bovine serum albumin $\mathrm{V}$ in Tris-buffered saline with $0.05 \%$ Tween-20 (TBST); pH 7.2]. Following three TBST washes for $5 \mathrm{~min}$, the membrane was incubated for $1 \mathrm{~h}$ at $37^{\circ} \mathrm{C}$ with FITC-conjugated goat anti-rabbit IgG antibody followed by coloration with 3,3-diaminobenzidine (DAB) tetrahydrochlorate using a DAB kit according to the manufacturer's instructions.

\section{Results}

Construction of expression plasmids. The PSCA gene and various structural domains of HSP70 were amplified by PCR with the respective primers. Then, the PSCA was cloned into pET21a(+) with the amino-terminus, carboxyl-terminus and overall length of HSP70, by enzyme digestion to construct the recombinant plasmids pET21-PSCA-HSPN, pET21-PSCA- 
A

B

$\mathrm{C}$

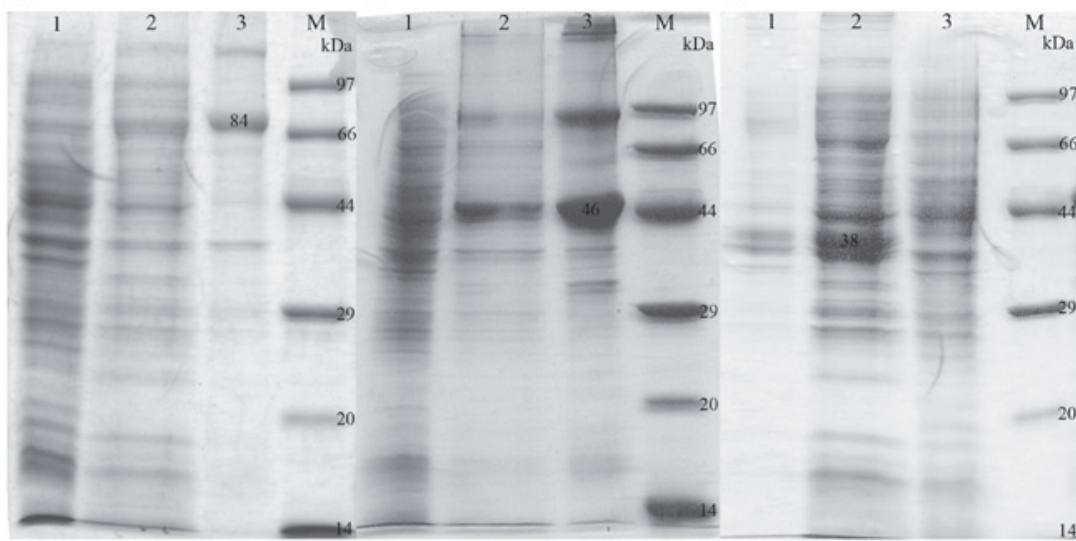

Figure 1. SDS-PAGE analysis of recombinant fusion proteins. (A) Lane 1, uninduced BL21(DE3)/pET21-PSCA-HSP; lane 2, induced BL21(DE3)/pET21-PSCAHSP, supernatant of the bacterial lysate; lane 3, induced BL21(DE3)/pET21-PSCA-HSP, pellet of the bacterial lysate; lane M, low molecular protein marker (97.2, 66.4, 44.3, 29.0, 20.1 and 14.3 kDa). (B) Lane 1, uninduced BL21(DE3)/pET21-PSCA-HSPN; lane 2, induced BL21(DE3)/ pET21-PSCA-HSPN, supernatant of the bacterial lysate; lane 3, induced BL21 (DE3)/pET21-PSCA-HSPN, pellet of the bacterial lysate; lane M, low molecular protein marker (97.2, 66.4, 44.3, 29.0, 20.1 and 14.3 kDa). (C) Lane 1, induced BL21(DE3)/pET21-PSCA-HSPC, pellet of the bacterial lysate; lane 2, induced BL21(DE3)/pET21PSCA-HSPC, supernatant of the bacterial lysate; lane 3, uninduced BL21(DE3)/pET21-PSCA-HSPC; lane M, low molecular protein marker (97.2, 66.4, 44.3, 29.0, 20.1 and 14.3 kDa). SDS-PAGE, sodium dodecyl sulfate-polyacrylamide gel electrophoresis; PSCA, prostate stem cell antigen; HSP, heat shock protein.

A

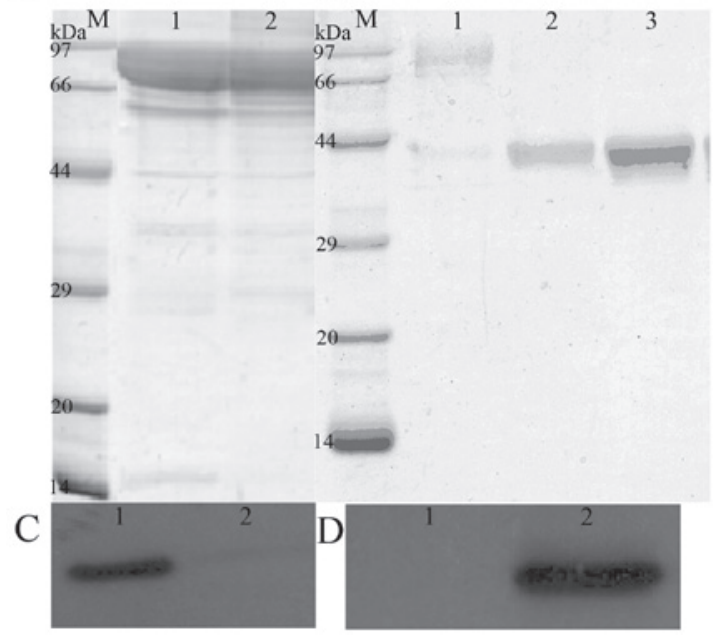

Figure 2. Purification and identification of recombinant fusion proteins. SDS-PAGE analysis of (A) PSCA-HSP and (B) SDS-PAGE analysis of PSCA-HSPC following purification with Ni-NTA resin, Phenyl-Sephrose Fast Flow and Superdex 75. (C) Identification of PSCA-HSP with western blotting. Lane 1, induced BL21(DE3)/pET21-PSCA-HSP; lane 2, uninduced BL21(DE3)/pET21-PSCA-HSP. (D) Identification of PSCA-HSPC with western blotting. Lane 1, uninduced BL21(DE3)/pET21-PSCA-HSPC; lane 2, induced BL21(DE3)/pET21-PSCA-HSPC. SDS-PAGE, sodium dodecyl sulfate-polyacrylamide gel electrophoresis; PSCA, prostate stem cell antigen; HSP, heat shock protein; Ni-NTA, nickel-nitrilotriacetic acid.

HSPC and pET21-PSCA-HSP, respectively. The recombinant plasmids containing the target gene sequences were further analyzed by restriction enzymatic pattern and finally confirmed to be in full accord with sequences issued by GenBank [PSCA cDNA (GenBank original accession no. AF04F3498) and HSP70 cDNA (no. NM005345)].

Expression of the recombinant fusion proteins. When the OD600 of the culture reached 1.2, E. coli cells harboring expression plasmids were treated with IPTG at a final concen- tration of $1 \mathrm{mM}$. The expression of proteins corresponding to the predicted size were induced in the presence of IPTG. The proteins PSCA-HSPC and PSCA-HSP expressed in E. coli were confirmed to exist in soluble form by SDS-PAGE analysis (Fig. 1).

Purification and identification of recombinant fusion proteins. The cell paste was collected following induction. Recombinant PSCA-HSPC (Fig. 2A) and PSCA-HSP (Fig. 2B) were purified from the supernatant of the bacteria lysate following treatment with a combination of Ni-NTA resin, Phenyl-Sepharose FF and HiLoad ${ }^{\mathrm{TM}} 26 / 60$ Superdex 75 . A purity up to $95 \%$ was achieved, as confirmed by SDS-PAGE.

Identification of recombinant fusion proteins with western blotting. After the proteins were transferred to the PVDF membranes, the PVDF membranes were treated successively with anti-PSCA monoclonal antibody and FITC-conjugated goat anti-rabbit IgG antibody. Western blotting confirmed the presence of PSCA (Fig. 2C and D).

\section{Discussion}

The PSCA gene encodes a 123 amino acid protein. Studies have identified a low-expression of the protein in normal prostate and a high-expression in prostate, bladder and pancreatic cancer $(10,11)$. Further studies demonstrated that the antibody of PSCA inhibits the growth of prostate cancer $(12,13)$. However, is is difficult to express PSCA in a soluble condition in E. coli due to its complicated spatial structure, which makes it difficult to develop the vaccine for prostate cancer treatment. Therefore, a method to enhance the soluble expression of PSCA and elevate the immunogenicity of PSCA must be established. If assisted with a suitable adjuvant molecule, the immunogenicity and solubility of PSCA may be enhanced, which may improve the potency of protein vaccines based on PSCA. 
The function of HSP70 in immunoadjuvant therapy has been identified and a number of studies have confirmed that vaccination with HSP70-peptide complexes and HSP70antigen fusion proteins reconstituted in vitro with genetic recombination elicit antitumor immune responses (14-17). In addition, according to the results of our pre-experiment, HSP70 increases the potency of a DNA vaccine based on PSCA with strong cellular and humoral immune responses, which inhibit the growth of PSCA-expressing tumors and prolong the survival time of vaccinated mice (18). Moreover, coupling antigens to the amino-terminus of HSP70 may induce stronger immune responses than coupling to the carboxyl-terminus (18). HSP70 has two functional domains: the carboxyl-terminus is a peptide-binding domain, which provokes the production of cytokines and the amino-terminus is the ATPase domain, which is not capable of provoking the generation of cytokines. This terminal inhibits the secretion of interleukin (IL)-10 and transforming growth factor (TGF)- $\beta$ (19). The antibody titres of anti-HSP70 or anti-HSPC generated by HSPC are lower compared with those generated by HSP70 (20).

In the present study, we presented evidence that human HSP70 enhances the solubility of PSCA. PSCA-HSPC and PSCA-HSP were successfully expressed to a high level in $E$. coli in soluble form and it is convenient for purification. PSCA-HSPN existed in insoluble form. Following three steps of purification, a purity of greater than $95 \%$ of the recombinant fusion proteins was obtained. Western blotting revealed that the PSCA and recombinant fusion proteins obtained via purification had the same immunological characteristics.

In conclusion, the present study confirmed the potency of human HSP70 as a molecular chaperone for a recombinant protein vaccine, which lays the foundation for the development of vaccines for prostate cancer and further clinical research.

\section{References}

1. Reiter RE, Gu Z, Watabe T, et al: Prostate stem cell antigen: A cell surface maker overexpressed in prostate cancer. Proc Natl Acad Sci USA 95: 1735-1740, 1998.

2. Gu Z, Thomas G, Yamashiro J, et al: Prostate stem cell antigen (PSCA) expression increases with high gleason score, advanced stage and bone metastasis in prostate cancer. Oncogene 19: 1288-1296, 2000.

3. Krupa M, Canamero M, Gomez CE, Najera JL, Gil J and Esteban M: Immunization with recombinant DNA and modified vaccinia virus Ankara (MVA) vectors delivering PSCA and STEAP1 antigens inhibits prostate cancer progression. Vaccine 29: 1504-1513, 2011.

4. Garcia-Hernandez Mde L, Gray A, Hubby B, Klinger OJ and Kast WM: Prostate stem cell antigen vaccination induces a long-term protective immune response against prostate cancer in the absence of autoimmunity. Cancer Res 68: 861-869, 2008.
5. Ahmad S, Casey G, Cronin M, Rajendran S, Sweeney P, Tangney M and O'Sullivan GC: Induction of effective antitumor response after mucosal bacterial vector mediated DNA vaccination with endogenous prostate cancer specific antigen. J Urol 186: 687-693, 2011.

6. Srivastava PK and Udono $\mathrm{H}$ : Heat shock protein-peptide complexes in cancer immunotherapy. Curr Opin Immunol 6: 728-732, 1994.

7. Srivastava PK, Udono H, Blachere NE and Li Z: Heat shock proteins transfer peptides during antigen processing and CTL priming. Immunogenetics 39: 93-98, 1994.

8. Blachere NE and Srivastava PK: Heat shock protein-based cancer vaccines and related thoughts on immunogenicity of human tumors. Semin Cancer Biol 6: 349-355, 1995.

9. Matsueda S, Kobayashi K, Nonaka Y, Noguchi M, Itoh K and Harada M: Identification of new prostate stem cell antigen-derived peptides immunogenic in HLA-A2(+) patients with hormonerefractory prostate cancer. Cancer Immunol Immunother 53: 479-489, 2004.

10. Bahrenberg G, Brauers A, Joost HG and Jakse G: PSCA expression is regulated by phorbol ester and cell adhesion in the bladder carcinoma cell line RT112. Cancer Lett 168: 37-43, 2001.

11. Argani P, Rosty C, Reiter RE, et al: Discovery of new markers of cancer through serial analysis of gene expression: prostate stem cell antigen is overexpressed in pancreatic adenocarcinoma. Cancer Res 61: 4320-4324, 2001.

12. Dannull J, Diener PA, Prikler L, et al: Prostate stem cell antigen is a promising candidate for immunotherapy of advanced prostate cancer. Cancer Res 60: 5522-5528, 2000.

13. Saffran DC, Raitano AB, Hubert RS, Witte ON, Reiter RE and Jakobovits A: Anti-PSCA mAbs inhibit tumor growth and metastasis formation and prolong the survival of mice bearing human prostate cancer xenografts. Proc Natl Acad Sci USA 98: 2658-2663, 2001

14. Blachere NE, Li Z, Chandawarkar RY, et al: Heat shock proteinpeptide complexes, reconstituted in vitro, elicit peptide-specific cytotoxic T lymphocyte response and tumor immunity. J Exp Med 186: 1315-1322, 1997.

15. Srivastava PK: Immunotherapy for human cancer using heat shock protein-peptide complexes. Curr Oncol Rep 7: 104-108, 2005.

16. Chu NR, Wu HB, Wu T, Boux LJ, Siegel MI and Mizzen LA: Immunotherapy of a human papillomavirus (HPV) type 16 E7-expressing tumour by administration of fusion protein comprising Mycobacterium bovis bacille Calmette-Guérin (BCG) hsp65 and HPV16 E7. Clin Exp Immunol 121: 216-225, 2000.

17. Liu DW, Tsao YP, Kung JT, Ding YA, Sytwu HK, Xiao X and Chen SL: Recombinant adeno-asseciated virus expressing human papilomavirus type 16 E7 peptide DNA fused with heat shock protein DNA as a potential vaccine for cervical cancer. J Virol 74: 2888-2894, 2000.

18. Zhang X, Yu C, Zhao J, et al: Vaccination with a DNA vaccine based on human PSCA and HSP70 adjuvant enhances the antigen-specific $\mathrm{CD}^{+} \mathrm{T}$-cell response and inhibits the $\mathrm{PSCA}^{+}$ tumors growth in mice. J Gene Med 9: 715-726, 2007.

19. Zhu X, Zhao X, Burkholder WF, Gragerov A, Ogata CM, Gottesman ME and Hendrickson WA: Structural analysis of substrate binding by the molecular chaperone DnaK. Science 272: 1606-1614, 1996.

20. Wang Y, Kelly CG, Singh M, McGowan EG, Carrara AS, Bergmeier LA and Lehner T: Stimulation of TH1-polarizing cytokines, $\mathrm{C}-\mathrm{C}$ chemokines, maturation of dendritic cells and adjuvant function by the peptide binding fragment of heat shock protein 70. J Immunol 169: 2422-2429, 2002. 